Research Article

\title{
The Quantum Effects Role on Weibel Instability Growth Rate in Dense Plasma
}

\author{
M. Mahdavi and F. Khodadadi Azadboni \\ Physics Department, University of Mazandaran, P.O. Box 47415-416, Babolsar, Iran \\ Correspondence should be addressed to M. Mahdavi; m.mahdavi@umz.ac.ir
}

Received 21 December 2014; Revised 4 March 2015; Accepted 4 March 2015

Academic Editor: Sally Seidel

Copyright (C) 2015 M. Mahdavi and F. Khodadadi Azadboni. This is an open access article distributed under the Creative Commons Attribution License, which permits unrestricted use, distribution, and reproduction in any medium, provided the original work is properly cited. The publication of this article was funded by SCOAP ${ }^{3}$.

\begin{abstract}
The Weibel instability is one of the basic plasma instabilities that plays an important role in stopping the hot electrons and energy deposition mechanism. In this paper, combined effect of the density gradient and quantum effects on Weibel instability growth rate is investigated. The results have shown that, by increasing the quantum parameter, for large wavelengths, the Weibel instability growth rate shrinks to zero. In the large wavelengths limit, the analysis shows that quantum effects and density gradient tend to stabilize the Weibel instability. The density perturbations have decreased the growth rate of Weibel instability in the near corona fuel, $\eta>0.1$. In the small wavelengths limit, for the density gradient, $\eta<0.1$, the tunneling quantum effects increase anisotropy in the phase space. The quantum tunneling effect leads to an unexpected increase in the Weibel instability growth rate.
\end{abstract}

\section{Introduction}

Quantum plasmas have attracted renowned attention in the recent years, due to, for example, the relevance of quantum effects in dense plasmas, very intense laser plasmas, and ultra-small semiconductor devices. The inclusion of quantum terms in the plasma fluid equations such as quantum diffraction effects, modified equations of state [1], and spin degrees of freedom leads to a variety of new physical phenomena [2]. Quantum plasmas can be found in laser-solid density plasma interaction experiments, laser-based inertial fusion, astrophysical and cosmological environments, and quantum diodes. In laser-dense plasma interaction, when the beam propagates in the plasma, it will induce a return current to keep current neutralization of the beam-plasma system, resulting in the well-known three instabilities; the twostream, current filamentation, and the Weibel instabilities [35]. The Weibel instability is one of the basic plasma instabilities that is driven by an anisotropic velocity distribution of plasma particles. Weibel instability arises from temperature anisotropy in the equilibrium distribution function and it is one of the fundamental instabilities of plasma physics. In more recent years, it has been the central concept in several instances, like fast ignition scenarios [6-15], particle acceleration, and magnetic field generation in astrophysical settings, collective non-Abelian Weibel instabilities in melting color glass condensates, covariant relativistic scenarios, electron-positron relativistic shocks, and laser heated plasmas. The Weibel instability plays an important role in stopping the hot electrons and energy deposition mechanism in the target. The energy deposition of a relativistic electron beam in a plasma can be managed through sufficiently steep plasma density gradients [16-24]. At the very initial stage of heating of the dense plasma, the electron temperature at the critical layer may be as low as a few $\mathrm{eV}$. The laser intensity is absorbed at a region with electron density of $0.02-1.0$ of the critical density. In this region the quantum effects are not totally negligible. For this purpose, the kinetic Wigner-Maxwell model which is the quantum counterpart of the Vlasov-Maxwell system has been used. The aim of this contribution is to get detailed information about the influence of quantum effects on Weibel instability in density gradient of dense plasma.

\section{Theoretical Model}

We consider linear transverse waves in a dense plasma composed of electrons and immobile ions, with $(\vec{k} \cdot \vec{E}=0)$, 
where $\vec{k}$ is the wave vector and $\vec{E}$ is the wave electric field. The propagation direction is the $z$-direction. The plasma will be heated only in the velocity dimension along the wave propagation direction, leading to a temperature anisotropy of the electron distribution that varies with the wave motion. Due to the density gradient of the target (the center is about $10^{4}$ denser than the border) that is in the $z$-direction, the beam-plasma interaction is collisionless near the relativistic electron beam-emitting region. In a dense laser created plasma, quantum effects must play an important role in the context of the Weibel instability. Following the standard procedure, one then obtains the general dispersion relation for the transverse waves of the Wigner-Maxwell system. The quantum dispersion relation for transverse waves is as given in

$$
\begin{aligned}
& \omega^{2}-c^{2} k^{2}-\omega_{p e}^{2} \\
&+\frac{m \omega_{p e}^{2}}{2 n_{0} \hbar} \int d v\left(\frac{v_{x}^{2}+v_{y}^{2}}{\omega-k v_{z}}\right) \\
& \cdot\left[f_{0}\left(\vec{v}+\frac{\hbar k}{2 m}\right)-f_{0}\left(\vec{v}-\frac{\hbar k}{2 m}\right)\right]=0,
\end{aligned}
$$

where $\omega_{p e}=\sqrt{4 \pi e^{2} n_{0} / m_{e} \gamma \eta}$ is the plasma frequency and $\eta(z, t)=\left[n_{0} / n_{e}(z, t)\right]^{2}$ is the density gradient in the $z$ direction. In general, in the absorption layer of fuel pellet, the thermal energy is much larger than both the potential energy and the Fermi energy, that is, the degeneracy parameter $\Theta=$ $\varepsilon_{F} / k_{B} T_{e}$ is much smaller than unity. Here e is the electron charge, $K_{B}$ is the Boltzmann constant, and $T_{e}$ is the electron temperature. This means that quantum degeneracy effects are negligibly small. Therefore, the analysis is restricted to nondegenerate plasmas, so that quantum effects due to spin and statistics are beyond the scope of this work. Therefore, the distribution of the electrons is described by anisotropic Maxwell-Boltzmann. The electron distribution function is

$$
\begin{aligned}
f_{0}(v)= & \frac{\sqrt{\eta} n_{0}}{T_{\|}^{1 / 2} T_{\perp}}\left(\frac{m_{e}}{2 \pi k_{B}}\right)^{3 / 2} \\
& \cdot \exp \left(-\frac{m_{e}}{2}\left[\frac{\left(v_{x}^{2}+v_{y}^{2}\right)}{k_{B} T_{\perp}}+\frac{\eta\left(v_{z}^{2}-v_{e z}^{2}\right)}{k_{B} T_{\|}}\right]\right),
\end{aligned}
$$

where $T_{\|}$and $T_{\perp}$ are related to the velocity dispersion in the direction perpendicular and in parallel to the $z$-axis, respectively, $\beta$ is $T_{\|} / T_{\perp}$, and $v_{e z}$ is the mean velocity of the particles. In application of these results to implosion of fuel pellet, density is [25]

$$
n_{e}(z, t)=n_{0} \exp \left(\frac{z}{l}+10\right)
$$

where $l$ is the density gradient scale length. The electron density and initial temperature of the plasma are taken to be $n_{0}=1 \times 10^{21} \mathrm{~cm}^{-3}$ and $T_{e 0}=500 \mathrm{keV}$, respectively. Considering spacetime dependence of the perturbations of the form exp $i(k z-\omega t)$, the dispersion equation for the Weibel transverse electromagnetic wave will obtain the following:

$$
\begin{aligned}
& \omega^{2}-k^{2} c^{2} \\
& \quad-\omega_{p e}^{2}\left(\sqrt{\eta}+\frac{m_{e} v_{t \|}}{2 \hbar k \beta}\left[\frac{2}{\sqrt{\pi}} \int_{-\infty}^{+\infty} d x\left(\frac{\exp \left(-x^{2}\right)}{(x-\xi)^{2}-R^{2}}\right)\right]\right) \\
& =0,
\end{aligned}
$$

where $\zeta=\left(\omega_{r}+i \delta\right) /\left(\sqrt{\eta} k v_{t \|}\right), \beta=T_{\|} / T_{\perp}, v_{t \|}=\left(2 k_{B} T_{\|} / m_{e}\right)^{1 / 2}$ is the electron thermal speed, and $R=\hbar k / 2 m_{e} v_{t \|}$ is a characteristic parameter representing the quantum diffraction effect. With considering the plasma dispersion function as

$$
Z(\xi)=\frac{1}{\sqrt{\pi}} \int_{-\infty}^{+\infty} d x\left(\frac{\exp \left(-x^{2}\right)}{x-\xi}\right),
$$

the dispersion relation will be corrected as follows:

$$
\begin{aligned}
\omega^{2} & -k^{2} c^{2} \\
& -\omega_{p e}^{2}\left(\sqrt{\eta}+\frac{m_{e} v_{t \|}}{2 \hbar k \beta}[Z(\xi+R)-Z(\xi-R)]\right)=0 .
\end{aligned}
$$

In limit large quantum effects, $R \gg 1$, and thus $R^{2} \gg|\xi|^{2}$, the dispersion relation simplifies to

$$
\omega^{2}-k^{2} c^{2}-\omega_{p e}^{2}\left(\sqrt{\eta}+\frac{1}{2 R^{2} \beta}\right)=0 .
$$

Specifically, for large quantum effects, there will be a purely growing wave only if

$$
\frac{T_{\perp}}{T_{\|}}>2 R^{2}\left(\left(\frac{c k}{\omega_{p e}}\right)^{2}+\sqrt{\eta}\right) .
$$

However, since the right-hand side of the last inequality is an increasing function of $R$ and $\sqrt{\eta}>0$, one can conclude that quantum effects play a stabilizing role. In limit small quantum effects, $R \ll 1$, and, small wavelengths, $\xi \ll 1$, the expansion of dispersion relation is as follows:

$$
\begin{aligned}
& \omega^{2}-k^{2} c^{2} \\
& -\omega_{p e}^{2}\left(\sqrt{\eta}+\frac{1}{\beta}[-1-\xi Z(\xi)\right. \\
& \left.\left.+\frac{R^{2}}{3}\left(2+3 \xi Z(\xi)-2 \xi^{2}-2 \xi^{3} Z(\xi)\right)\right]\right) \\
& =0 \text {. }
\end{aligned}
$$

And, in the plasma dispersion function, $Z(\xi) \cong i \sqrt{\pi}$, the dispersion relation is given by

$$
\omega^{2}-k^{2} c^{2}-\omega_{p e}^{2}\left(\sqrt{\eta}+\frac{1}{\beta}\left[-1-i \xi \sqrt{\pi}+\frac{2 R^{2}}{3}\right]\right)=0 .
$$


The growth rate of the Weibel instability $\delta\left(\omega=\omega_{r}+i \delta\right.$, where $\omega_{r}$ and $\delta$ are both real numbers) is determined by

$$
\delta=\frac{\sqrt{\eta} k v_{t \|}}{\sqrt{\pi}}\left[1-\frac{2 R^{2}}{3}-\left(\beta\left(\sqrt{\eta}+\left(\frac{k c}{\omega_{p e}}\right)^{2}\right)\right)\right] .
$$

There can be instability $(\delta>0)$, provided that there is also sufficient temperature anisotropy:

$$
\frac{T_{\perp}}{T_{\|}}>\frac{\sqrt{\eta}}{\left(1-2 R^{2} / 3\right)} \cong \sqrt{\eta}\left(1+\frac{2 R^{2}}{3}\right) .
$$

This condition implies that growing instability at the edge of fuel pellet needs extra anisotropy, due to quantum effects. But with the advance towards the center of the fuel pellet and decreasing the density gradient, required temperature anisotropy decreases. The unstable wave numbers of the Weibel instability are equal to

$$
k_{\text {cut }}=\frac{\omega_{p e}}{c}\left(\frac{T_{\perp}}{T_{\|}}\left(1-\frac{2 R^{2}}{3}\right)-\sqrt{\eta}\right)^{1 / 2},
$$

where the allowable unstable wave numbers, $k_{\text {cut }}$, occur for a smaller range for increasing quantum parameter, $R$. In limit small quantum effects, $R \ll 1$, and, large wavelengths, $\xi \gg$ 1 , using the expansion $Z(\xi) \cong-1 / \xi-1 / 2 \xi^{3}$, the dispersion relation is given by

$$
\omega^{2}-k^{2} c^{2}-\omega_{p e}^{2}\left(\sqrt{\eta}+\frac{1}{2 \beta \xi^{2}}\left(1-R^{2}\right)\right)=0 .
$$

Using definition thermal speed and $\xi$, the dispersion relation reduces to

$$
\omega^{2}-k^{2} c^{2}-\omega_{p e}^{2}\left(\sqrt{\eta}+\frac{\eta k^{2} k_{B} T_{\perp}}{m_{e} \omega^{2}}\left(1-R^{2}\right)\right)=0 .
$$

For $|\omega| \ll c k$, the growth rate of the Weibel instability is given by

$$
\delta=\sqrt{\frac{\eta k^{2} k_{B} T_{\perp}\left(1-R^{2}\right)}{m_{e}\left(\sqrt{\eta}+\left(k c / \omega_{p e}\right)^{2}\right)}}=0 .
$$

It is shown that purely growing waves are excited when

$$
\frac{T_{\perp}}{T_{\|}} \gg \eta\left(1-R^{2}\right)
$$

One sees that for increasing quantum effects there is the need for extra temperature anisotropy. Due to wave-particle spreading and tunneling, quantum effects tend to enhance the dispersion of particles in phase space [26]. Therefore, the effectively temperature anisotropy decreases, so that the temperature anisotropy has to be greater to produce the same instability results as in classical plasma.

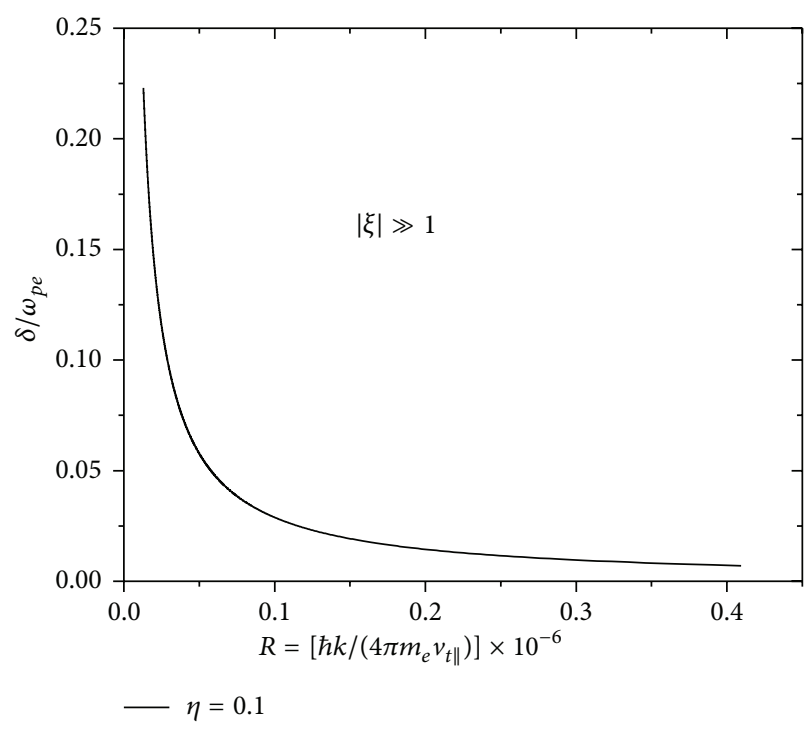

FIgURE 1: The normalized growth rate of the Weibel instability, $\delta / \omega_{p e}$, as a function of quantum parameter $R=\hbar k / 2 m_{e} v_{t \|}$, for $\eta=0.1$, and the large wavelengths.

\section{Results and Discussions}

As expected and displayed in Figure 1, for increasing quantum effects (larger values of $R=\hbar k / 2 m_{e} v_{t \|}$ ), the instability occurs with smaller maximal growth rates and unstable wave numbers shrink to zero. In Figure 2, the normalized growth rate, $\delta / \omega_{p e}$, is plotted as a function of the normalized wavenumber, $k c / \omega_{p e}$, for different values of the density gradient, $\eta$. Generally, as discussed by Schaefer-Rolffs and Tautz [23], the quantum effects without density gradient produce smaller Weibel instability growth rate and smaller ranges for $\eta>0.1$, in the case of equilibria with distribution functions anisotropic in temperature. Considering density gradient in fuel pellet in limit large wavelengths, quantum effects lead to increasing growth rates and smaller ranges for unstable wave numbers (Figure 2(a)). Also, for the larger values of density gradient, $\eta>0.1$, the maximum growth rate is quickly decreased and the instability occurs with smaller maximal wave numbers. As is shown in Figure 2(b), in limit small wavelengths for $\eta<0.1$, quantum effects lead to an unexpected increase in the rate of growth. In interaction short-pulse laser-dense plasma of fuel, due to wave-particle spreading and tunneling, quantum effects tend to enhance the dispersion of particles in phase space. This corresponds to increases of temperature anisotropy and increases of Weibel instability rate. Figure 3 shows the growth rate of Weibel instability in terms of several values of density gradient, small wavelengths, and large wavelengths. For fixed quantum parameter, in transmission of relativistic electron beam from the side of the fusion pellet to its core and decreasing gradient density (the quantity $g$ will have a variation range between zero and one, $0.0001<\eta<1$ ), the thermal spread of the energetic electrons reduces the growth rate of Weibel instability. For $\eta=0.1$, in limit small wavelengths, $\xi \ll$ 1 , the instability growth rate and unstable wavenumber are 


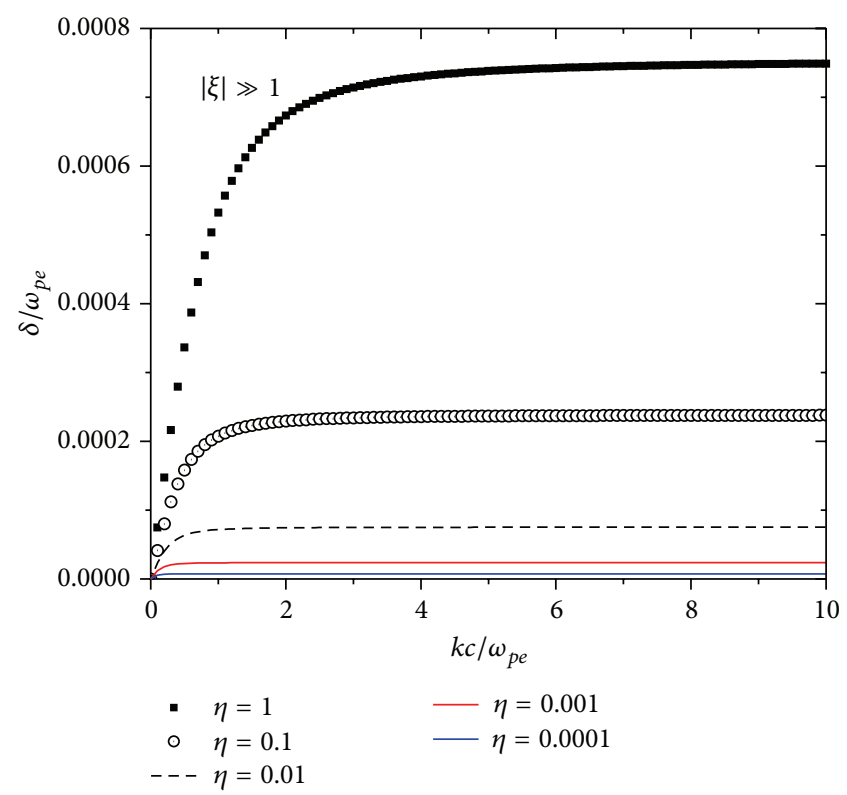

(a)

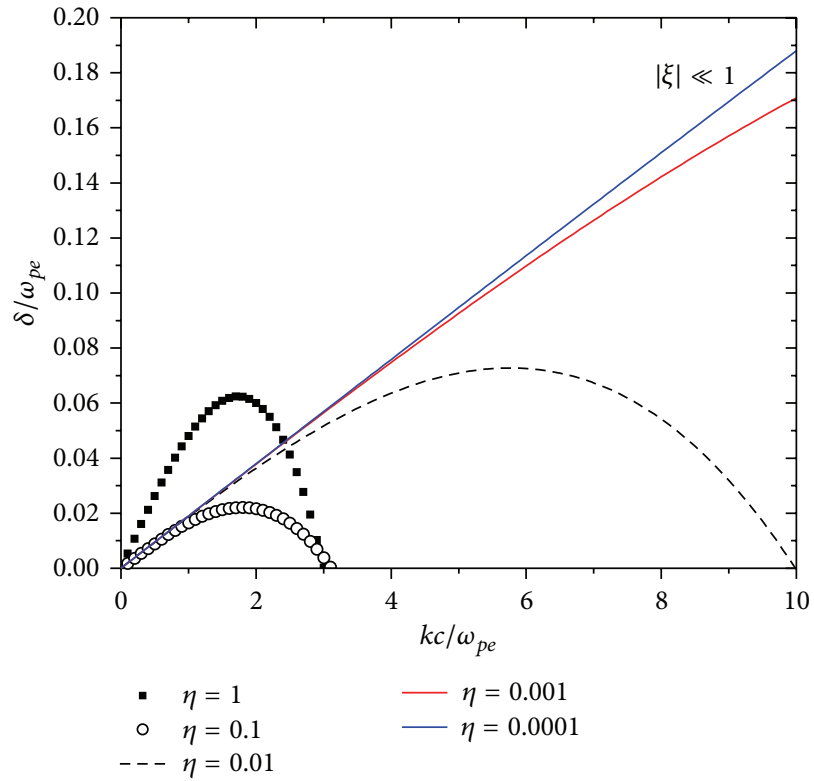

(b)

FIGURE 2: The growth rate of the Weibel instability, $\delta / \omega_{p e}$, as a function of wavenumber, $k c / \omega_{p e}$, for the fixed density gradient, $\eta$, (a) for the large wavelengths, (b) for the small wavelengths.

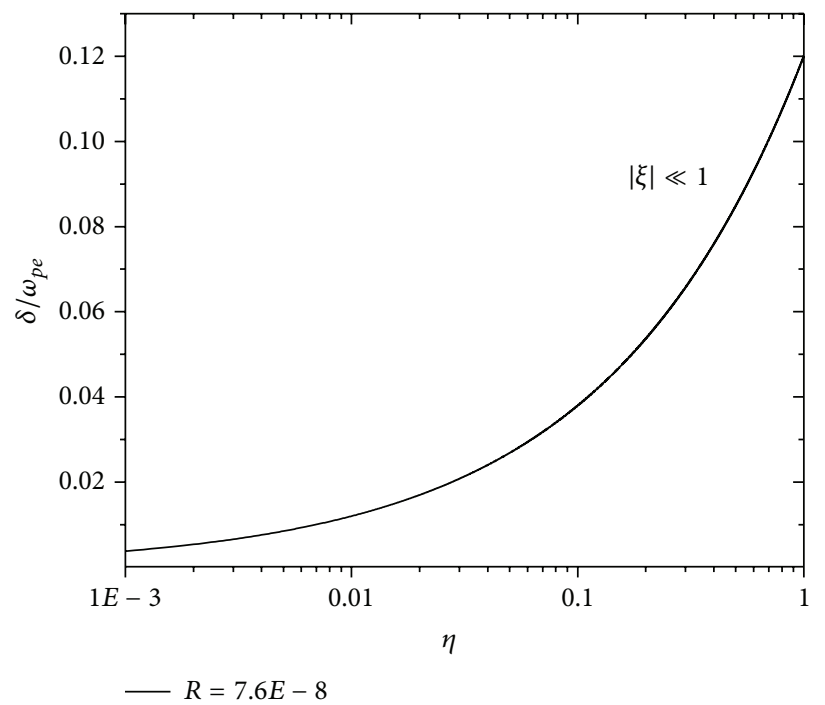

FIgURE 3: The normalized growth rate of the Weibel instability, $\delta / \omega_{p e}$, as a function of density gradient, $\eta$, for the fixed quantum parameter $R=\hbar k / 2 m_{e} v_{t \|}$.

nonlinear proportional to quantum parameter (Figure 4(a)), but, in limit large wavelengths, $\xi \gg 1$, plasma has different treatments (Figure 4(b)).

\section{Conclusions}

The Weibel instability is one of the basic plasma instabilities that is driven by an anisotropic velocity distribution of plasma particles. The study of this kind of instability is very important as it plays a very crucial role in heat deposition process from laser to dense plasma. In the interaction laser-dense plasma, a fast electron beam which makes its way to the target core generates at the critical density. The fast electron beam traveling through a very important density gradient, since the critical density where the laser deposits its energy, is smaller than the core density by four orders. Therefore, it is important to evaluate the effect of a density gradient upon the growth rate of Weibel instability. Calculations show that the Weibel instability growth rate is dependent on the density gradient of target and the quantum effects such as spin and tunneling, so increasing the quantum parameter and decreasing the density 


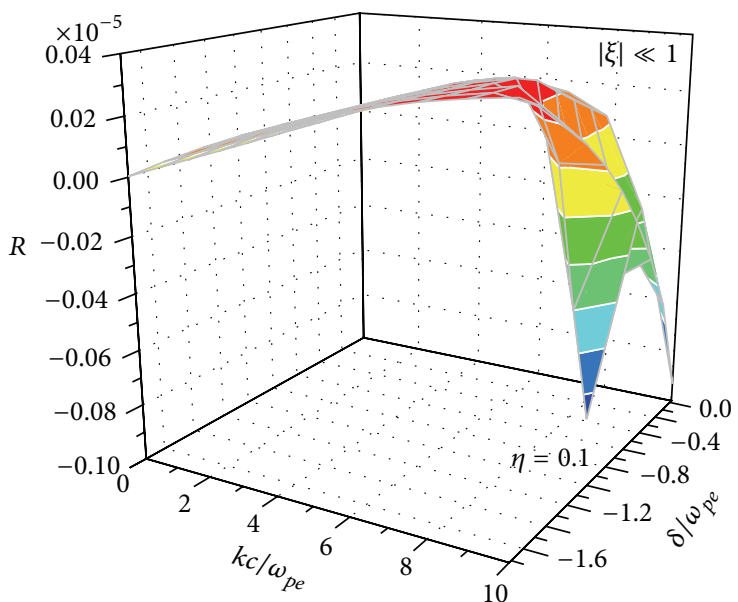

(a)

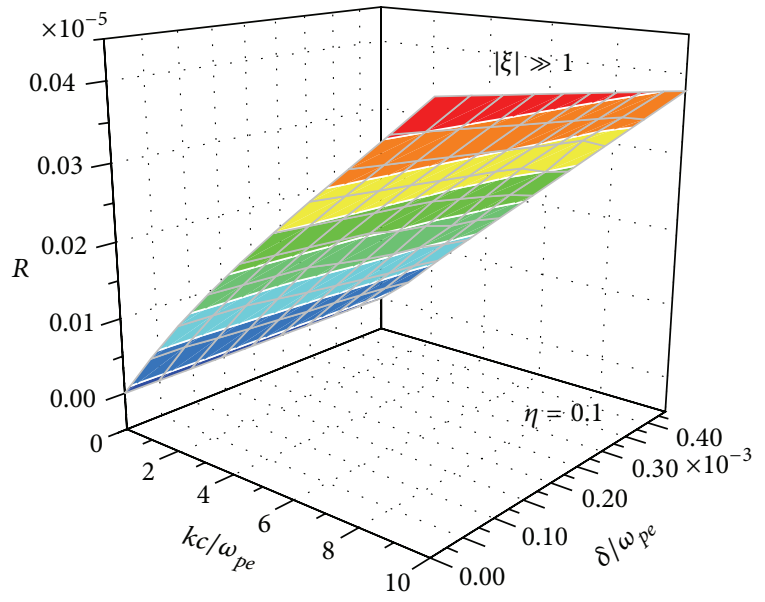

(b)

FIGURE 4: The growth rate of the Weibel instability, $\delta / \omega_{p e}$, as a function of wavenumber, $k c / \omega_{p e}$, and quantum parameter, $R$, for the fixed density gradient, $\eta$, (a) for the small wavelengths, (b) for the large wavelengths.

gradient in large wavelength will decrease the instability. The maximum growth rate of Weibel instability and wavenumber decreases by traveling the relativistic electron beam towards the target center. There is a critical scale length $\eta_{c}=0.1$, under which the growth rate starts to increase as $\eta$ decreases. For $\eta<\eta_{c}$, this could be attributed to sometimes quantum effects; for example, tunneling can give unexpected enhancement of Weibel instability growth rate and unstable wavenumber.

\section{Conflict of Interests}

The authors declare that there is no conflict of interests regarding the publication of this paper.

\section{References}

[1] F. Haas, G. Manfredi, and M. R. Feix, "Multistream model for quantum plasmas," Physical Review E, vol. 62, no. 2, pp. 27632772, 2000.

[2] M. Marklund and G. Brodin, "Dynamics of spin-1/2 quantum plasmas," Physical Review Letters, vol. 98, no. 2, Article ID 025001, 4 pages, 2007.

[3] S. Y. Gus'kov, "Thermonuclear gain and parameters of fast ignition ICF-targets," Laser and Particle Beams, vol. 23, no. 2, pp. 255-260, 2005.

[4] P. Velarde, F. Ogando, S. Eliezer, J. M. Martínez-Val, J. M. Perlado, and M. Murakami, "Comparison between jet collision and shell impact concepts for fast ignition," Laser and Particle Beams, vol. 23, no. 1, pp. 43-46, 2005.

[5] C. T. Zhou and X. T. He, "Influence of a large oblique incident angle on energetic protons accelerated from solid-density plasmas by ultraintense laser pulses," Applied Physics Letters, vol. 90, no. 3, Article ID 031503, 3 pages, 2007.

[6] C. T. Zhou and X. T. He, "Intense laser-driven energetic proton beams from solid density targets," Optics Letters, vol. 32, no. 16, pp. 2444-2446, 2007.
[7] M. Mahdavi and F. Khodadadi Azadboni, "The effect of degeneracy parameter on Weibel instability in dense plasma," Physics of Plasmas, vol. 20, no. 12, Article ID 122708, 2013.

[8] M. Mahdavi and H. Khanzadeh, "Collisional effect on the Weibel instability with the bi-Maxwellian distribution function," Physics of Plasmas, vol. 20, no. 5, Article ID 052114, 2013.

[9] R. Jung, J. Osterholz, K. Löwenbrück et al., "Study of electronbeam propagation through preionized dense foam plasmas," Physical Review Letters, vol. 94, no. 19, Article ID 195001, 2005.

[10] K. V. Starikov and C. Deutsch, "Stopping of relativistic electrons in a partially degenerate electron fluid," Physical Review E, vol. 71, Article ID 026407, 2005.

[11] J. R. Davies, "Electric and magnetic field generation and target heating by laser-generated fast electrons," Physical Review E, vol. 68, Article ID 056404, 2003.

[12] V. M. Malkin and N. J. Fisch, "Collective deceleration of relativistic electrons precisely in the core of an inertial-fusion target," Physical Review Letters, vol. 89, no. 12, Article ID 125004, 4 pages, 2002.

[13] M. Mahdavi and F. K. Azadboni, "The effect of density gradient on the growth rate of relativistic Weibel instability," Physics of Plasmas, vol. 21, no. 2, Article ID 022707, 2014.

[14] R. C. Fadanelli, J. F. Dias, and M. Behar, "Coulomb heating behavior of fast light diclusters thorough the $\mathrm{Si}\langle 110\rangle$ direction: influence of the mean charge state," The European Physical Journal D, vol. 68, pp. 1-5, 2014.

[15] A. Bret and C. Deutsch, "Density gradient effects on beam plasma linear instabilities for fast ignition scenario," Laser and Particle Beams, vol. 24, no. 2, pp. 269-273, 2006.

[16] R. J. Mason, "Heating mechanisms in short-pulse laser-driven cone targets," Physical Review Letters, vol. 96, Article ID 035001, 4 pages, 2006.

[17] M. Tzoufras, C. Ren, F. Tsung et al., "Space-charge effects in the current-filamentation or weibel instability," Physical Review Letters, vol. 96, Article ID 105002, 2 pages, 2006.

[18] M. V. Medvedev and A. Leob, "Generation of magnetic fields in the relativistic shock of gamma-ray burst sources," The Astrophysical Journal, vol. 526, no. 2, pp. 697-706, 1999. 
[19] K. O. Mikaelian and J. D. Lindl, "Density gradients to reduce fluid instabilities in multishell inertial-confinement-fusion targets," Physical Review A, vol. 29, no. 1, pp. 290-296, 1984.

[20] H. Ren, Z. Wu, J. Cao, C. Dong, and P. K. Chu, "Density gradient effects on the magnetorotational instability," Plasma Physics and Controlled Fusion, vol. 53, no. 3, Article ID 035012, 2011.

[21] A. Bret and C. Deutsch, "Beam plasma electromagnetic instabilities in a smooth density gradient: application to the fast ignition scenario," Physics of Plasmas, vol. 12, no. 10, Article ID 102702, 2005.

[22] J. Pétri and J. G. Kirk, "Numerical solution of the linear dispersion relation in a relativistic pair plasma," Plasma Physics and Controlled Fusion, vol. 49, no. 3, p. 297, 2007.

[23] U. Schaefer-Rolffs and R. C. Tautz, "The relativistic kinetic weibel instability: comparison of different distribution functions," Physics of Plasmas, vol. 15, no. 6, Article ID 062105, 2008.

[24] M. Honda, J. Meyer-ter-Vehn, and A. Pukhov, "Collective stopping and ion heating in relativistic-electron-beam transport for fast ignition," Physical Review Letters, vol. 85, no. 10, pp. 2128-2131, 2000.

[25] W. P. Leemans, C. E. Clayton, W. B. Mori et al., "Experiments and simulations of tunnel-ionized plasmas," Physical Review A, vol. 46, no. 2, pp. 1091-1105, 1992.

[26] F. Haas, "Quantum Weibel instability," Physics of Plasmas, vol. 15, no. 2, Article ID 022104, 6 pages, 2008. 

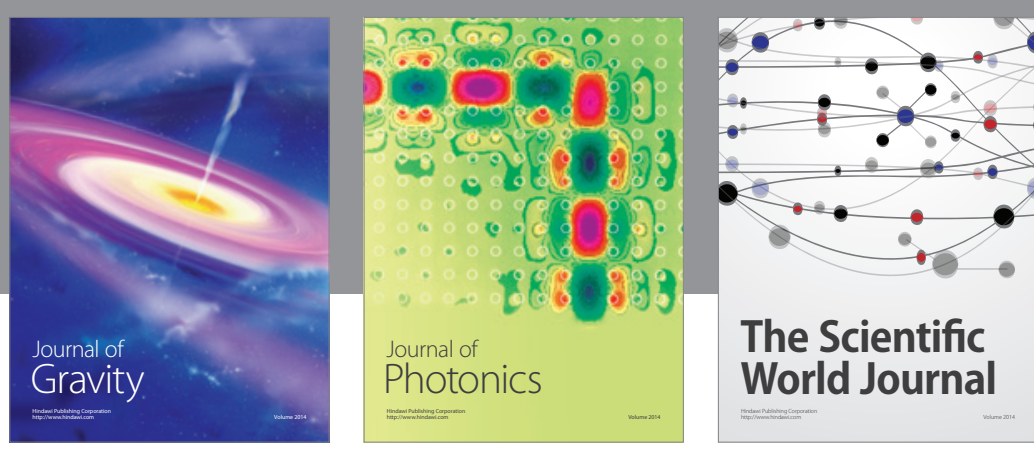

The Scientific World Journal
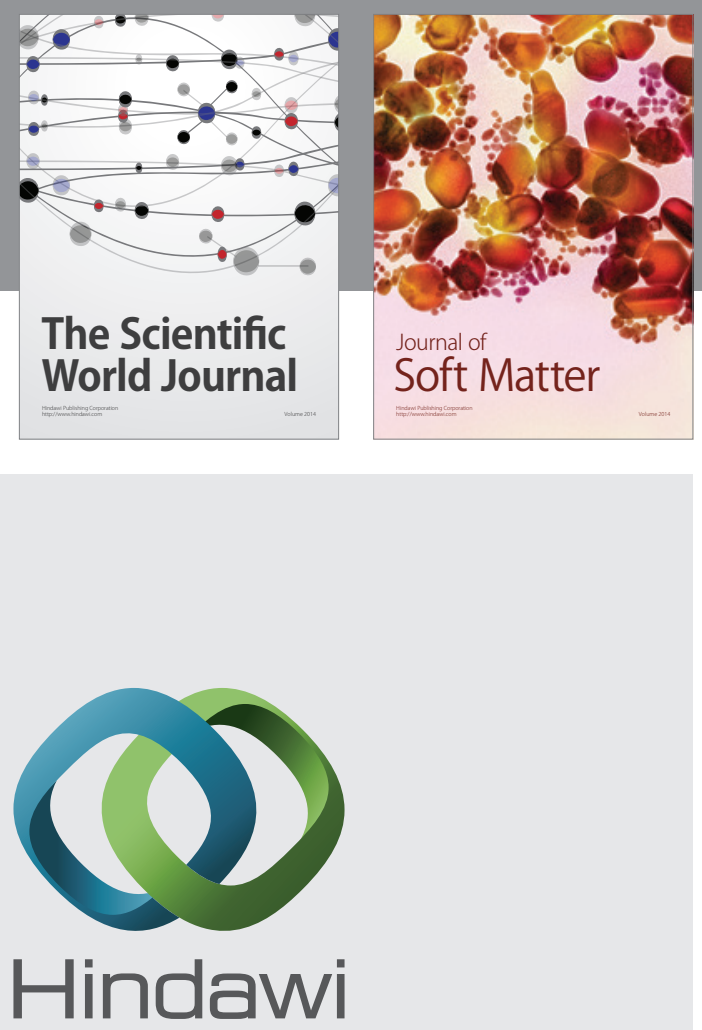

Submit your manuscripts at

http://www.hindawi.com

nternational Journal of

Statistical Mechanics
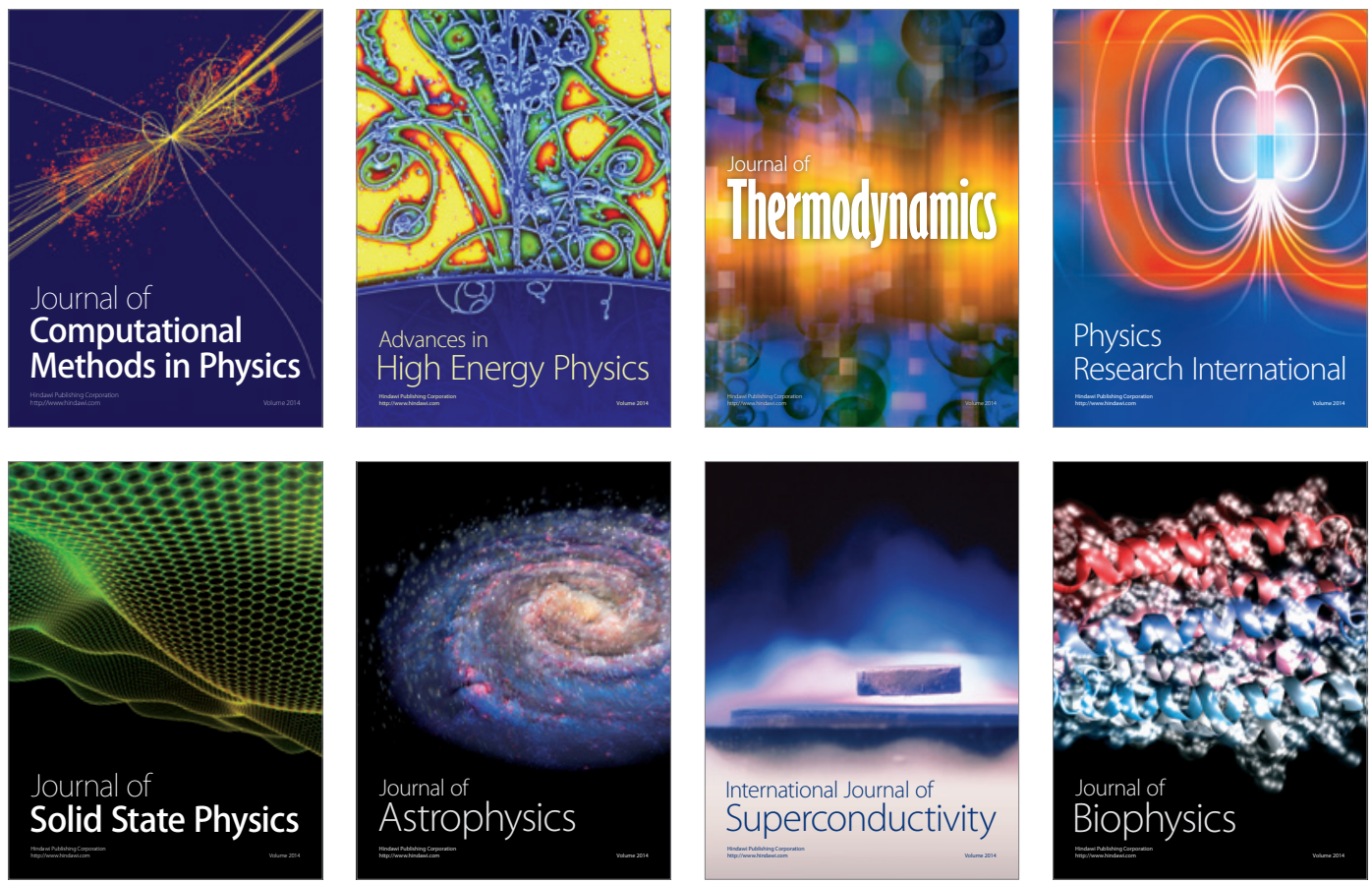
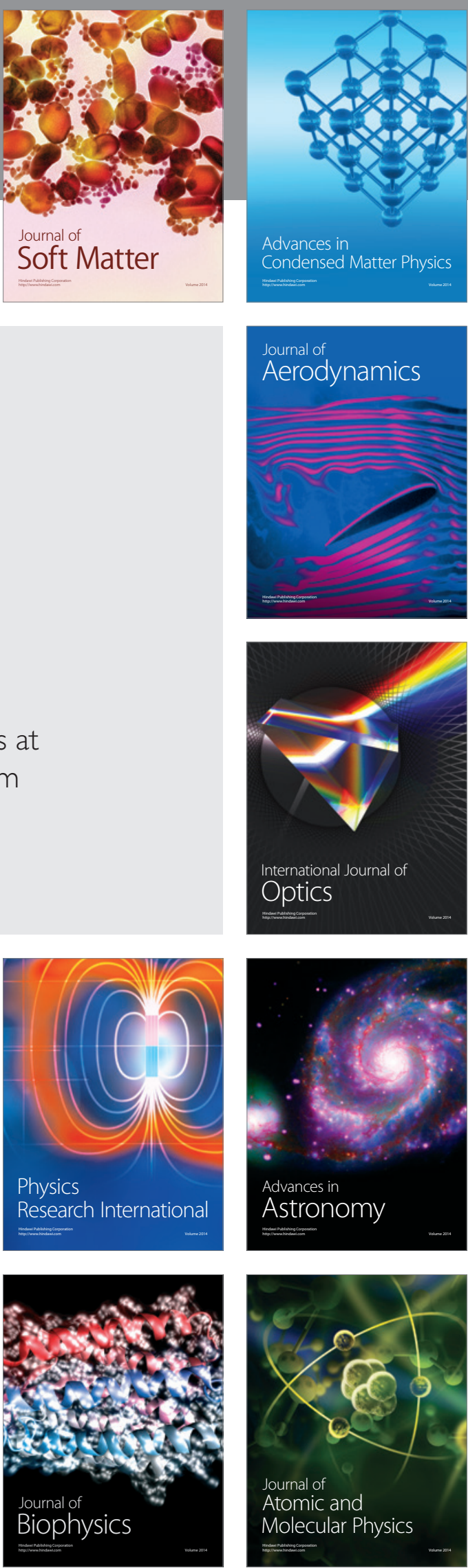